\title{
Medikamentöse Therapie bei Frauen - Was gilt es zu beachten?
}

\author{
Ursula Ravens
}

Online publiziert: 20. März 2013

(C) Springer-Verlag Berlin Heidelberg 2013

\begin{abstract}
Zusammenfassung Frauen und Männer unterscheiden sich in ihren Reaktionen gegenüber Medikamenten. Geschlechtsabhängige pharmakokinetische Unterschiede betreffen vorwiegend die Metabolisierungsgeschwindigkeit und Verteilung von Arzneistoffen, meist sind die Plasmaspiegel bei Frauen höher als bei Männern. Das Risiko für Frauen, unerwünschte Arzneimittelwirkungen zu erleiden, ist signifikant größer als für Männer. Die Geschlechtsabhängigkeit der medikamentösen Therapie hat vielerlei Gründe, sie weist deutlich auf die Notwendigkeit hin, dass die biologischen Unterschiede zwischen Männern und Frauen bei der Pharmakotherapie gezielt untersucht und möglichst auch in den Leitlinien berücksichtigt werden müssen.
\end{abstract}

Schlüsselwörter Geschlechtsabhängigkeit ·

Medikamentöse Therapie · Pharmakokinetik ·

Pharmakodynamik $\cdot$ Risiko für unerwünschte

Arzneimittelwirkungen (UAW)

\begin{abstract}
Women and men exhibit different reactions to drugs. Sex-based pharmacokinetic differences relate mainly to the rate of biotransformation and to the distribution of drugs. Women have a significantly larger risk for adverse drug reactions than men. Sex-dependence of drug therapy is a multifactorial problem clearly demonstrating the necessity for specific investigations into the biological differences between women and men concerning drug therapy. Known differences should be included into guide line recommendations.
\end{abstract}

Prof. Dr. med. U. Ravens $(\bowtie)$

Institut für Pharmakologie und Toxikologie, Medizinische

Fakultät Carl Gustav Carus, Technische Universität Dresden,

Fetscherstraße 74, 01307 Dresden, Deutschland

E-Mail: ravens@rcs.urz.tu-dresden.de
Keywords Sex differences - Drug therapy · Pharmacokinetics - Pharmacodynamics · Risk for adverse drug reactions (ADR)

\section{Einleitung}

Genetische Unterschiede zwischen einzelnen Individuen schlagen sich sowohl in der Symptomatik und dem Verlauf von Krankheiten als auch in der Ansprechbarkeit auf therapeutische Maßnahmen nieder. Mit der Entschlüsselung des menschlichen Genoms ist die Forderung nach personalisierter Medizin unüberhörbar geworden und gleichzeitig in den Bereich des Möglichen gerückt. Vor diesem allgemeinen Hintergrund verwundert die Tatsache, dass offensichtliche biologisch (,,sex“) und sozial bedingte (,gender") Geschlechtsunterschiede zwischen Männern und Frauen nur in Ausnahmefällen Berücksichtigung bei Diagnostik und Therapie von Krankheiten finden. Ein Grund hierfür mag die unzureichende Datenlage sein, denn trotz Anmahnungen der großen Arzneimittelzulassungsbehörden wie der Food and Drug Administration (FDA) oder der European Medicines Agency (EMA) sind Frauen bei klinischen Arzneimittelprüfungen auch heute noch eindeutig unterrepräsentiert [1-3]. Das Wissen über die Wirksamkeit neuer Medikamente bei Frauen muss als lückenhaft bezeichnet werden. Dennoch werden in der Regel nach der Zulassung keine generellen Einschränkungen für die Anwendung bei Frauen ausgesprochen, während für den Fall einer Schwangerschaft $90 \%$ aller neu zugelassenen Medikamente als kontraindiziert eingestuft werden, obwohl hier ebenfalls keine Daten vorliegen [2].

In den vergangenen zwei Jahrzehnten sind zwar deutlich mehr Frauen als Männer für klinische Studien, die vom National Institute of Health der USA finanziert wurden, rek- 
rutiert worden, dies ist aber nur auf einige wenige frauenspezifische Projekte zurückzuführen [3], wie z. B. Studien zum Mamma-, Zervix- und Uteruskarzinom, die Women's Health Studie zu Aspirin und Vitamin E bei kardiovaskulären Erkrankungen und Krebs [4] und die große Langzeitstudie über Frauen in der Postmenopause durch die Women's Health Initiative [5].

Datenbanken zur Arzneimittelsicherheit zeigen, dass unerwünschte Arzneimittelwirkungen (UAW) häufiger bei Frauen als bei Männern auftreten [6, 7]. Als Gründe hierfür sind anzuführen, dass Frauen und Männer sich hinsichtlich der Pharmakokinetik, d. h. wie Arzneimittel sich im Körper verteilen und wieder unwirksam gemacht werden, und in geschlechtsspezifischen Reaktionen auf Arzneimittel (Pharmakodynamik), aber auch hinsichtlich der Menge an eingenommenen Medikamenten unterscheiden [8,9].

In der vorliegenden Übersicht über medikamentöse Therapien bei Frauen soll zunächst auf die wichtigsten geschlechtsbedingten Unterschiede hinsichtlich Pharmakokinetik und Pharmakodynamik eingegangen werden, gefolgt von einigen therapeutischen Beispielen, bei denen frauenspezifische Besonderheiten bekannt sind. Abschließend werden Möglichkeiten zur Verbesserung der medikamentösen Therapie von Frauen aufgezeigt.

\section{Pharmakokinetik}

Die Dosierung eines Medikaments und seine pharmakokinetischen Eigenschaften bestimmen die Konzentration am Wirkort und somit die Wirkstärke. Geschlechtsbezogene Unterschiede in der Pharmakokinetik [8,9] können daher die Effektivität einer medikamentösen Therapie entscheidend beeinflussen. Das gilt in besonderem Maße für Medikamente mit enger therapeutischer Breite, bei denen bereits geringfügig höhere oder $\mathrm{zu}$ niedrige Konzentrationen $\mathrm{zu}$ toxischen Wirkungen bzw. zu einem Therapieversagen führen können.

Aufnahme Bei Frauen ist die Magenpassage verzögert, sodass Medikamente nach oraler Applikation etwas langsamer resorbiert werden als bei Männern. Allerdings resorbieren Frauen Alkohol rascher als Männer, weil die Alkoholdehydrogenase im Magen weniger aktiv ist.

Verteilung Frauen sind im Durchschnitt kleiner und wiegen weniger als Männer. Sie haben einen höheren Anteil von Fettgewebe am Gesamtkörpervolumen, dagegen sind der intrazelluläre, extrazelluläre und Gesamtwassergehalt niedriger als bei Männern [10]. Aufgrund dieser anatomischen Unterschiede besitzen Frauen ein anderes Verteilungsvolumen für Pharmaka. So ist das Verteilungsvolumen für viele Arzneimittel wie z. B. Diazepam bei Frauen höher, für andere dagegen wie z. B. Alkohol oder Alprazolam kleiner als bei Männern $[9,11]$.

Trotz der offensichtlichen Unterschiede bleibt das Körpergewicht bei der Dosierung im klinischen Alltag meist unberücksichtigt, sodass Frauen in Relation zu ihrem Körpergewicht fast immer eine zu hohe Dosis erhalten. In einer Bioäquivalenzstudie der FDA lag die Fläche unter der Kurve für Arzneimittel, bei denen ein geschlechtsspezifischer Unterschied festgestellt worden war, bei Frauen um 20-88 \% höher als bei Männern, wenn nicht um das Körpergewicht korrigiert wurde [12]. Aber auch nach Korrektur um das Körpergewicht waren die maximale Konzentration $\mathrm{C}_{\max }$ und die Fläche unter der Kurve bei $15 \%$ der geprüften Arzneimittel zwischen Frauen und Männern signifikant verschieden. Möglicherweise ist dies eine der Ursachen für eine erhöhte Inzidenz von UAWs.

Metabolismus Die wichtigsten Arzneimittel abbauenden Enzyme in der Leber sind die Isoenzyme von Cytochrom P450, Uridindiphosphatglucoronosyltransferase (UGT), N-Acetyltransferase (NAT) und Methyltransferase (MT). Für viele dieser Enzyme sind Polymorphismen mit zum Teil erheblichen Aktivitätsunterschieden bekannt. Allerdings gibt es zurzeit keine Hinweise auf eine Assoziation zwischen Geschlecht und den bekannten Polymorphismen in den Arzneimittel abbauenden Enzymen.

Die Expression und Aktivität einzelner Isoenzyme von Cytochrom P450 kann bei Frauen und Männern sehr verschieden sein [10, 11]. Das Isoenzym CYP3A4 ist für die Biotransformation der Hälfte aller Arzneistoffe, einschließlich von Sexualhormonen und Glukokortikoiden verantwortlich. Frauen haben eine vermehrte Expression und Aktivität von CYP3A4 [13], dadurch ist die Metabolisierungsgeschwindigkeit z. B. für Verapamil oder Nifedipin um etwa $20 \%$ höher als bei Männern [14, 15]. Werden mehrere von CYP3A4 abgebaute Medikamente gleichzeitig gegeben, kann es durch eine gegenseitige Abbauhemmung zum Anstieg der Plasmaspiegel kommen, die das Risiko für unerwünschte Wirkungen erhöhen.

Andere P450-Isoenzyme haben eine geringere Aktivität bei Frauen als bei Männern (Tab. 1). $\beta$-Rezeptorenblocker werden vornehmlich über das CYP2D6 abgebaut. Nach Gabe einer Standarddosis eines $\beta$-Blockers weisen Frauen deutlich höhere Plasmakonzentrationen als Männer auf [16-18].

Die Enzyme der Kopplungsreaktionen UGT, NAT und MT zeigen entweder keine geschlechtsabhängigen Aktivitätsunterschiede oder eine niedrigere Aktivität bei Frauen als bei Männern (Tab. 1). Dies trägt zur erhöhten Toxizität verschiedener Zytostatika bei Frauen bei [9]. 
Tab. 1 Geschlechtsbezogene Unterschiede in der Aktivität von Arzneimittel abbauenden Enzymen, zusammengestellt nach [10, 11]

\begin{tabular}{|c|c|c|c|}
\hline Isoenzym & Aktivität & Modellsubstanz & Biotransformierte Arzneimittel \\
\hline CYP $3 \mathrm{~A} 4$ & $\mathrm{~F}>\mathrm{M}$ & $\begin{array}{l}\text { Cortisol, Dapson, } \\
\text { Erythromycin }\end{array}$ & $\begin{array}{l}\text { Alfentanil, Alprazolam, Astemizol, Atorvastatin, Carbamazepin, Ciclosporin, Cisaprid, } \\
\text { Clarithromycin, Cortisol, Cyclophosphamid, Diazepam, Diltiazem, Erythromycin, Estradiol, } \\
\text { Fentanyl, Indinavir, Itraconazol, Ketoconazol, Lidocain, Lovastatin, Midazolam, Nifedipin, } \\
\text { Nimodipine, Nisoldipin, Chinidin, Ritonavir, Simvastatin, Tacrolimus, Tamoxifen, Tirilazid, } \\
\text { Troglitazon, Verapamil, Vinblastine, Vincristin }\end{array}$ \\
\hline CYP 2A6 & $\mathrm{F}>\mathrm{M}$ & & Verschiedene \\
\hline CYP 2 B6 & $\mathrm{F}>\mathrm{M}$ & - & Alfentanil, Cyclophosphamid, Ketamin, Propofol, Tamoxifen \\
\hline CYP2D6 & $\mathrm{F} \geq \mathrm{M}$ & Debrisoquin, Spartein & $\begin{array}{l}\text { Clomipramin, Codein, Desipramin, Encainid, Flecainid, Fluoxetin, Haloperidol, Hydrocodon, } \\
\text { Imipramin, Metoprolol, Mexiletin, Paroxetin, Pheformin, Propafenon, Propranolol, Sertralin, } \\
\text { Testosterol, Timolol }\end{array}$ \\
\hline CYP 1A2 & $\mathrm{F}<\mathrm{M}$ & Coffein, Nicotin & Clozapin, Paracetamol, Theophyllin \\
\hline CYP $2 \mathrm{E} 1$ & $\mathrm{~F}<\mathrm{M}$ & Clorzoxazon & - \\
\hline UGT & $\mathrm{F}<\mathrm{M}$ & Coffein & Clofibrinsäure, Diflusinal, Ibuprofen, Mycophenolat-Mofetil, Paracetamol, Zidovudin \\
\hline MT & $\mathrm{F}<\mathrm{M}$ & $\begin{array}{l}\text { Noradrenalin, } \\
\text { Adrenalin }\end{array}$ & Azathioprin, Dopamin, Levodopa, Mercaptopurin, Thioguanin \\
\hline CYP 2C19 & $\mathrm{F} \leq \mathrm{M}$ & Diazepam & $\begin{array}{l}\text { Celecoxib, Citalopram, Hexobarbital, Imipramin, Irbesartan, Lansoprazol, Methylphenobarbital, } \\
\text { Omeprazol, Piroxicam, Propranolol (teilweise) }\end{array}$ \\
\hline CYP 2 C9 & $\mathrm{F}=\mathrm{M}$ & Dapson & $\begin{array}{l}\text { Fluvastatin, Glipizide, Ibuprofen, Irbesartan, Losartan, Nelfinavir, Phenytoin, Piroxicam, } \\
(\text { S)-Warfarin, Tolbutamide }\end{array}$ \\
\hline NAT & $\mathrm{F}=\mathrm{M}$ & Coffein, Dapson & Katecholamin-Derivate, Hydralazin, Isoniazid, Mercaptopurin \\
\hline
\end{tabular}

Pharmaka mit kardiovaskulärer Indikation im weitesten Sinne sind kursiv hervorgehoben.

CYP Cytochrom P450, UGT „uridine diphosphate glucoronosyltransferase“, MT Methyltransferase, NAT N-Acetyltransferase, F Frauen, $M$ Männer

Renale Ausscheidung Die glomeruläre Filtrationsrate ist bei Frauen etwa 10\% niedriger als bei Männern [19, 20]. Für Digoxin konnte bei Frauen eine 12-14\% niedrigere renale Clearance nachgewiesen werden. Dies erklärt die in der Nachanalyse der Digitalis-Investigation-Group- (DIG-) Studie gefundenen höheren Plasmaspiegel von Frauen [21]. Auch Antibiotika, die unverändert renal eliminiert werden, wie z. B. Vancomycin oder Ceftazidin, erreichen wegen der verminderten renalen Clearance bei Frauen höhere Plasmakonzentrationen als bei Männern [11]. Das Transporterprotein P-Glykoprotein fördert die renale Clearance. Geschlechtsspezifische Unterschiede sind nicht klar erkennbar.

\section{Pharmakodynamik}

Geschlechtsbasierte Unterschiede in der Arzneimittelwirkung (Pharmakodynamik) sind eher selten und oftmals nur schwer von pharmakokinetischen Effekten zu trennen. Neben vielen anderen Faktoren ist ein genomischer Einfluss von Sexualhormonen auf die Expression von Rezeptoren als eine Ursache für die geschlechtsspezifische Ansprechbarkeit auf Arzneimittel denkbar. Allerdings gibt es nur wenig Information über die Variabilität von Arzneimittelwirkungen in Abhängigkeit vom menstruellen Zyklus [22].
Therapeutische Wirkungen Bei Frauen können vergleichbare analgetische Wirkungen mit geringeren Dosen an Opiaten als bei Männern erzielt werden [23, 24]. Dies wird auf eine vermehrte Expression von Opioid-Rezeptoren zurückgeführt [25]. Für die klinische Wirksamkeit von Antidepressiva bei chronischer Depression sind ebenfalls signifikante Geschlechtsunterschiede nachgewiesen worden [26]: Während Frauen besser auf den selektiven SerotoninWiederaufnahmeblocker Sertralin als auf das trizyklische Antidepressivum Imipramin ansprachen, waren bei Männern die Verhältnisse umgekehrt. Als naheliegende Erklärung kann der stimulierende Einfluss von Östrogenen auf die serotonerge Aktivität herangezogen werden. Möglich ist aber auch, dass Frauen eher atypische Symptome der Depression zeigen, die besser auf Serotonin-Wiederaufnahmeblocker als auf trizyklische Antidepressiva ansprechen [26].

In der kardiovaskulären Therapie hat die Post-hoc-Analyse der DIG-Studie unterschiedliche Effekte von Digoxin bei Männern und Frauen ergeben: Die Digoxin-Behandlung einer Herzinsuffizienz war bei Frauen mit einem erhöhten Mortalitätsrisiko im Vergleich zu Placebo assoziiert, nicht dagegen bei Männern [27, 28]. Der Mechanismus der Interaktion zwischen weiblichem Geschlecht und Digoxin-Therapie konnte jedoch nicht identifiziert werden.

In den beiden großen Acetylsalicylsäure- (ASS-)Studien zur primären Prävention kardiovaskulärer Ereignisse wurden eindeutige geschlechtsspezifische Unterschiede in 
der Art der Prävention gefunden [29]. Während ASS bei Männern das Myokardinfarktrisiko um 44\% senkte, war es bei Frauen in dieser Hinsicht unwirksam. Auf das Schlaganfallrisiko hatte ASS bei Männern keinen Einfluss, während dieses bei Frauen um 17\% gesenkt wurde. Weder bei Männern noch bei Frauen senkte ASS die Gesamtmortalität kardiovaskulärer Erkrankungen [30, 31]. Als Erklärung für die einander entgegengesetzten Ergebnisse aus den ASSStudien bei Männern und Frauen wurden anatomische, physiologische und funktionelle Unterschiede im HerzKreislauf-System, ein unterschiedlicher Hormonstatus, aber auch unterschiedliche Zeitpunkte für die Durchführung der beiden Studien diskutiert [29].

Unerwünschte Arzneimittelwirkungen, Toxizität Nicht nur die therapeutische Wirksamkeit, sondern auch die Toxizität von Arzneimitteln kann zwischen Frauen und Männern erheblich variieren [32]. Einer der häufigsten Gründe für die Marktrücknahme von neuen Arzneimitteln ist das Auftreten von Lebertoxizität, wobei $74 \%$ von akutem Leberversagen durch Medikamente bei Frauen auftritt [33].

Frauen haben ein um den Faktor 1,5-1,7 höheres Risiko UAWs zu entwickeln als Männer [6, 34]. Die Gründe hierfür sind nicht eindeutig. Frauen erhalten aufgrund ihrer im Durchschnitt geringeren Körpergröße eine höhere Dosierung in Bezug auf ihr Körpergewicht und eliminieren Medikamente oftmals langsamer, sodass sie höhere Arzneimittelkonzentrationen aufweisen. Neben diesen pharmakokinetischen Gesichtspunkten werden aber Frauen in der Regel mehr Medikamente verschrieben als Männern, sie sind oft zuverlässiger in der Einnahme der verschriebenen Medikamente, und sie mögen auch eher als Männer bereit sein, über ihre Beschwerden zu sprechen. Selbst wenn diese Faktoren rechnerisch berücksichtigt werden, blieb die beobachtete Inzidenz von UAWs in einer großen britischen Studie bei Frauen pro 10.000 Patientenmonate signifikant erhöht [35].

Viele Beispiele lassen sich für die deutlichen Geschlechtsunterschiede in der Verträglichkeit und der Art der unerwünschten Wirkungen anführen. Arzneimittelinduzierte Torsade-de-pointes-Arrhythmien (TdP) sind häufiger bei Frauen als bei Männern, dies gilt sowohl für Antiarrhythmika als auch für Pharmaka mit nicht kardialer Indikation [36, 37] (s. auch Odening K in dieser Ausgabe). Das erhöhte TdP-Risiko bei Frauen wird zum einen auf die längere frequenzkorrigierte QT-Zeit im EKG (QTc), zum anderen auf die bei Frauen größere QTc-Verlängerung durch die von den auslösenden Medikamenten hervorgerufene KaliumKanal-Blockade zurückgeführt [38, 39]. Der für ACE-Hemmer charakteristische trockene Reizhusten tritt bei Frauen häufiger als bei Männern auf [17]. Bei leitliniengerechter Therapie eines akuten Koronarsyndroms ohne ST-StreckenAnhebung (non-ST-elevation) haben Frauen ein etwa dop- pelt so hohes Risiko schwerwiegende Blutungen zu erleiden als Männer, und dieses Risiko wird noch verstärkt, wenn sie zusätzlich mit Glykoprotein-IIb/IIIa- (GPIIb/IIIa-)Inhibitoren behandelt werden [40]. Interessanterweise wären $25 \%$ dieser Blutungen vermeidbar, wenn die Dosis der GPIIb/ IIIa-Inhibitoren bei Frauen an ihr niedrigeres Körpergewicht und ihre im Durchschnitt schlechtere Nierenfunktion angepasst worden wäre [40].

Unerwünschte Arzneimittelwirkungen stellen ein ernst $\mathrm{zu}$ nehmendes Gesundheitsproblem dar, das regelmäBig zu Krankenhauseinweisungen, Morbidität oder sogar Tod führt [41-43]. Die Inzidenz von Krankenhauseinweisungen wegen UAWs liegt zwischen 3 und $6 \%$ aller Hospitalisierungen [42, 43]. Je nach untersuchter Population standen schwerwiegende UAWs an vierter bis siebter Stelle der häufigsten Todesursachen [42, 44]. In den Niederlanden sind Rodenburg et al. kürzlich der Frage nach geschlechtsspezifischen Unterschieden für Krankenhauseinweisungen wegen UAWs in einer die gesamte nationale Bevölkerung umfassenden Analyse nachgegangen [45]. Es wurden nahezu 10 Mio. Krankenhauseinweisungen über einen Zeitraum von 6 Jahren (2000-2005) ausgewertet. Geschlechtsbezogene Unterschiede im Risiko für eine Einweisung wegen UAWs fanden sich für folgende Arzneimittelgruppen, nämlich kardiovaskuläre, neurologische, antineoplastische und immunsuppressive Medikamente, Antirheumatika, Antikoagulanzien, Salicylate, Steroide und Antibiotika. Insgesamt wurden mehr Frauen als Männer wegen UAWs hospitalisiert, allerdings kehrte sich das Verhältnis bei einigen Medikamenten um, wenn die Anzahl der Verschreibungen berücksichtigt wurde. Unter den kardiovaskulären Arzneimitteln führten Diuretika und Saluretika am häufigsten zur Hospitalisation. Im Vergleich zu Männern hatten Frauen ein mehr als 5-faches Risiko wegen Hypoosmolarität oder Hyponatriämie und ein mehr als 3-faches Risiko wegen Hypokaliämie eingewiesen zu werden, und dieses erhöhte Risiko blieb auch nach Korrektur um die größere Verschreibungshäufigkeit bestehen. Interessanterweise traten Synkopen oder Kollapse nach Vasodilatatoren oder Hypovolämien nach Saluretika häufiger bei Männern als bei Frauen auf [45].

\section{Was ist zu tun?}

Die genannten Beispiele für unterschiedliche pharmakologische Reaktionen bei Frauen und Männern zeigen, dass geschlechtsspezifische Besonderheiten für die individuelle Arzneimittelsicherheit eine Rolle spielen, wenngleich unser Wissen darüber noch in den Anfängen steht. Eine Verbesserung dieser Situation erfordert gezielte Maßnahmen. 
- Arzneimittelzulassende Behörden müssen darauf bestehen, dass Frauen in alle zulassungsrelevanten klinischen Studien für neue Arzneimittel paritätisch einbezogen werden. Bei Nichteinhalten dieser Bedingung ist eine ausdrückliche Begründung erforderlich.

- Klinische Studien müssen konsequent auf geschlechtsspezifische Unterschiede hin analysiert werden.

- Bekanntes und neues Wissen über unterschiedliche Arzneimittelreaktionen zwischen Frauen und Männern sollte sich in den Leitlinien für Diagnostik und Therapie verschiedener Krankheitsbilder niederschlagen [46]. Dies gilt ganz besonders auch für die Empfehlungen von Leitlinien zur medikamentösen Therapie von Frauen.

Autorenerklärung Zwischen der Autorin dieses Beitrags und Herstellern von Arzneimitteln, die die besprochenen Wirkstoffe enthalten, bestehen keine finanziellen Verbindungen.

Acknowledgments This article is part of a supplement sponsored by Lilly Deutschland GmbH and Daiichi Sankyo Deutschland GmbH.

\section{Literatur}

1. Harris DJ, Douglas PS (2000) Enrollment of women in cardiovascular clinical trials funded by the national heart, lung, and blood institute. N Engl J Med 343:475-480

2. Schmucker DL, Vesell ES (1993) Underrepresentation of women in clinical drug trials. Clin Pharmacol Ther 54:11-15

3. Kim AM, Tingen CM, Woodruff TK (2010) Sex bias in trials and treatment must end. Nature 465:688-689

4. Lee IM, Cook NR, Gaziano JM, Gordon D, Ridker PM, Manson JE, Hennekens CH, Buring JE (2005) Vitamin E in the primary prevention of cardiovascular disease and cancer: the women's health study: a randomized controlled trial. JAMA 294:56-65

5. Rossouw JE, Anderson GL, Prentice RL, LaCroix AZ, Kooperberg C, Stefanick ML, Jackson RD, Beresford SA, Howard BV, Johnson KC, Kotchen JM, Ockene J (2002) Risks and benefits of estrogen plus progestin in healthy postmenopausal women: principal results from the women's health initiative randomized controlled trial. JAMA 288:321-333

6. Evans RS, Lloyd JF, Stoddard GJ, Nebeker JR, Samore MH (2005) Risk factors for adverse drug events: a 10-year analysis. Ann Pharmacother 39:1161-1168

7. Rademaker M (2001) Do women have more adverse drug reactions? Am J Clin Dermatol 2:349-351

8. Anderson GD (2005) Sex and racial differences in pharmacological response: where is the evidence? Pharmacogenetics, pharmacokinetics, and pharmacodynamics. J Womens Health (Larchmt) 14:19-29

9. Schwartz JB (2003) The influence of sex on pharmacokinetics. Clin Pharmacokinet 42:107-121

10. Soldin OP, Mattison DR (2009) Sex differences in pharmacokinetics and pharmacodynamics. Clin Pharmacokinet 48:143-157

11. Anderson GD (2008) Gender differences in pharmacological response. Int Rev Neurobiol 83:1-10
12. Chen ML, Lee SC, Ng MJ, Schuirmann DJ, Lesko LJ, Williams RL (2000) Pharmacokinetic analysis of bioequivalence trials: implications for sex-related issues in clinical pharmacology and biopharmaceutics. Clin Pharmacol Ther 68:510-521

13. Wolbold R, Klein K, Burk O, Nussler AK, Neuhaus P, Eichelbaum M, Schwab M, Zanger UM (2003) Sex is a major determinant of CYP3A4 expression in human liver. Hepatology 38:978-988

14. Krecic-Shepard ME, Barnas CR, Slimko J, Jones MP, Schwartz JB (2000) Gender-specific effects on verapamil pharmacokinetics and pharmacodynamics in humans. J Clin Pharmacol 40:219-230

15. Krecic-Shepard ME, Park K, Barnas C, Slimko J, Kerwin DR, Schwartz JB (2000) Race and sex influence clearance of nifedipine: results of a population study. Clin Pharmacol Ther 68:130-142

16. Luzier AB, Killian A, Wilton JH, Wilson MF, Forrest A, Kazierad DJ (1999) Gender-related effects on metoprolol pharmacokinetics and pharmacodynamics in healthy volunteers. Clin Pharmacol Ther 66:594-601

17. Thurmann PA (2005) Gender-related differences in pharmacokinetics and pharmacodynamics. Bundesgesundheitsblatt Gesundheitsforschung Gesundheitsschutz 48:536-540

18. Walle T, Byington RP, Furberg CD, McIntyre KM, Vokonas PS (1985) Biologic determinants of propranolol disposition: results from 1308 patients in the beta-blocker heart attack trial. Clin Pharmacol Ther 38:509-518

19. Meibohm B, Beierle I, Derendorf H (2002) How important are gender differences in pharmacokinetics? Clin Pharmacokinet 41:329-342

20. Schwartz JB (2007) The current state of knowledge on age, sex, and their interactions on clinical pharmacology. Clin Pharmacol Ther 82:87-96

21. Rathore SS, Curtis JP, Wang Y, Bristow MR, Krumholz HM (2003) Association of serum digoxin concentration and outcomes in patients with heart failure. JAMA 289:871-878

22. Kashuba AD, Nafziger AN (1998) Physiological changes during the menstrual cycle and their effects on the pharmacokinetics and pharmacodynamics of drugs. Clin Pharmacokinet 34:203-218

23. Craft RM (2003) Sex differences in opioid analgesia: „from mouse to man". Clin J Pain 19:175-186

24. Pleym H, Spigset O, Kharasch ED, Dale O (2003) Gender differences in drug effects: implications for anesthesiologists. Acta Anaesthesiol Scand 47:241-259

25. Zubieta JK, Smith YR, Bueller JA, Xu Y, Kilbourn MR, Jewett DM, Meyer CR, Koeppe RA, Stohler CS (2002) mu-opioid receptor-mediated antinociceptive responses differ in men and women. J Neurosci 22:5100-5107

26. Kornstein SG, Schatzberg AF, Thase ME, Yonkers KA, McCullough JP, Keitner GI, Gelenberg AJ, Davis SM, Harrison WM, Keller MB (2000) Gender differences in treatment response to sertraline versus imipramine in chronic depression. Am J Psychiatry 157:1445-1452

27. Rathore SS, Wang Y, Krumholz HM (2002) Sex-based differences in the effect of digoxin for the treatment of heart failure. $\mathrm{N}$ Engl J Med 347:1403-1411

28. Blaustein MP, Robinson SW, Gottlieb SS, Balke CW, Hamlyn JM (2003) Sex, digitalis, and the sodium pump. Mol Interv 3:68-72, 50

29. Levin RI (2005) The puzzle of aspirin and sex. N Engl J Med 352:1366-1368

30. Final report on the aspirin component of the ongoing Physicians' Health Study. Steering Committee of the Physicians' Health Study Research Group (1989) N Engl J Med 321:129-135

31. Ridker PM, Cook NR, Lee IM, Gordon D, Gaziano JM, Manson JE, Hennekens CH, Buring JE (2005) A randomized trial of lowdose aspirin in the primary prevention of cardiovascular disease in women. N Engl J Med 352:1293-1304 
32. Nicolson TJ, Mellor HR, Roberts RR (2010) Gender differences in drug toxicity. Trends Pharmacol Sci 31:108-114

33. Miller MA (2001) Gender-based differences in the toxicity of pharmaceuticals - the food and drug administration's perspective. Int J Toxicol 20:149-152

34. Zopf Y, Rabe C, Neubert A, Gassmann KG, Rascher W, Hahn EG, Brune K, Dormann H (2008) Women encounter ADRs more often than do men. Eur J Clin Pharmacol 64:999-1004

35. Martin RM, Biswas PN, Freemantle SN, Pearce GL, Mann RD (1998) Age and sex distribution of suspected adverse drug reactions to newly marketed drugs in general practice in England: analysis of 48 cohort studies. Br J Clin Pharmacol 46:505-511

36. Drici MD, Clement N (2001) Is gender a risk factor for adverse drug reactions? The example of drug-induced long QT syndrome. Drug Saf 24:575-585

37. Makkar RR, Fromm BS, Steinman RT, Meissner MD, Lehmann MH (1993) Female gender as a risk factor for torsades de pointes associated with cardiovascular drugs. JAMA 270:2590-2597

38. Coker SJ (2008) Drugs for men and women - how important is gender as a risk factor for TdP? Pharmacol Ther 119:186-194

39. Jonsson MK, Vos MA, Duker G, Demolombe S, van Veen TA (2010) Gender disparity in cardiac electrophysiology: implications for cardiac safety pharmacology. Pharmacol Ther 127:9-18

40. Alexander KP, Chen AY, Newby LK, Schwartz JB, Redberg RF, Hochman JS, Roe MT, Gibler WB, Ohman EM, Peterson ED (2006) Sex differences in major bleeding with glycoprotein IIb/ IIIa inhibitors: results from the CRUSADE (Can Rapid risk stratification of Unstable angina patients Suppress ADverse outcomes with Early implementation of the ACC/AHA guidelines) initiative. Circulation 114:1380-1387
41. Beijer HJ, de Blaey CJ (2002) Hospitalisations caused by adverse drug reactions (ADR): a meta-analysis of observational studies. Pharm World Sci 24:46-54

42. Lazarou J, Pomeranz BH, Corey PN (1998) Incidence of adverse drug reactions in hospitalized patients: a meta-analysis of prospective studies. JAMA 279:1200-1205

43. Pirmohamed M, James S, Meakin S, Green C, Scott AK, Walley TJ, Farrar K, Park BK, Breckenridge AM (2004) Adverse drug reactions as cause of admission to hospital: prospective analysis of 18,820 patients. BMJ 329:15-19

44. Wester K, Jonsson AK, Spigset O, Druid H, Hagg S (2008) Incidence of fatal adverse drug reactions: a population based study. Br J Clin Pharmacol 65:573-579

45. Rodenburg EM, Stricker BH, Visser LE (2011) Sex-related differences in hospital admissions attributed to adverse drug reactions in the Netherlands. Br J Clin Pharmacol 71:95-104

46. Weinbrenner S, Lonnfors S, Babitsch B (2010) Gender: new methodological approaches in guideline development. Z Evid Fortbild Qual Gesundhwes 104:547-553 